The

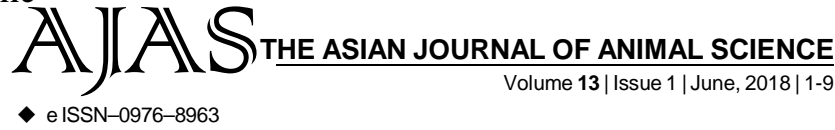

DOI : 10.15740/HAS/TAJAS/13.1/1-9

Visit us |WWW researchjournal.co in "SA

RESEARCH ARTICLE........

\title{
Process optimization for propagation of Lactobacillus acidophilus NCFM LYO 10D
}

Mahadevaiah, H. M. Jayaprakasha and K.B. Suresha

Author for Corresponding -

\section{K.B. Suresha}

AICRP on Post-Harvest

Engineering and Technology,

University of Agricultural

Sciences, Bengaluru

(Karnataka) India

See end of the article for

Coopted authors'
ABSTRACT...... The present work was under taken to optimize the propagation of $L$. acidophilus in different mediums for blending into the functional weaning food. A strain of probiotics namely Lactobacillus acidophilus NCFM LYO 10D was propagated in whey medium using different prebiotics such as honey, carrot and tomato (CT) juice and whey protein hydrolysate (WPH). The study revealed that 1.5 per cent inoculum and incubation for a period of $15 \mathrm{~h}$ incubation at $37^{\circ} \mathrm{C}$ is optimum for obtaining the maximum effect in decreasing $\mathrm{pH}$ of whey medium. The addition of honey has significant effect in decreasing the $\mathrm{pH}$ of whey medium upto 2 per cent honey. Supplementation of honey was also found to have significant effect on the viable count of La-N. The extent of increase in viable count was found be significant upto 2 per cent level. The supplementation of CT juice at a level of 4 per cent was found to have significant effect in decreasing $\mathrm{pH}$ and highest viable count. WPH addition has significant effect increase in acidity and decrease $\mathrm{pH}$ of whey medium upto 1.5 per cent level. The viable count of La-N significantly increased upon supplementation of WPH. It was concluded that best combination for propogation of La-N in whey medium was found to be supplementation of honey upto 2 per cent, CT juice at a level of 4 per cent, WPH addition upto 1.5 per cent in whey medium and adding 1.5 per cent inoculum and incubating for $15 \mathrm{~h}$ at $37^{\circ} \mathrm{C}$.

KEY WORDS...... Propagation, Lactobacillus acidophilus, NCFM LYO 10D

HOW TO CITE THIS ARTICLE - Mahadevaiah, Jayaprakasha, H.M. and Suresha, K.B. (2018). Process optimization for propagation of Lactobacillus acidophilus NCFM LYO 10D. Asian J. Animal Sci., 13(1): 1-9. DOI : 10.15740/HAS/TAJAS/13.1/1-9. Copyright@ 2018:HindAgri-Horticultural Society.

ARTICLE CHRONICLE - Received : 03.03.2018; Revised : 10.05.2018; Accepted : 21.05.2018 